\title{
A comprehensive study of the link between star-formation history and X-ray source populations in the SMC
}

\author{
Vallia Antoniou ${ }^{1,2}$, Andreas Zezas ${ }^{1}$ and Despina Hatzidimitriou ${ }^{2}$ \\ ${ }^{1}$ Harvard-Smithsonian Center for Astrophysics, 60 Garden St., Cambridge, MA 02138, USA \\ email: vantoniou@cfa.harvard.edu \\ ${ }^{2}$ Physics Department, University of Crete, P.O. Box 2208, GR-710 03, Heraklion, Crete, Greece
}

\begin{abstract}
Using Chandra, XMM-Newton and optical photometric catalogs we study the young $\mathrm{X}$-ray binary (XRB) populations of the Small Magellanic Cloud (SMC). We find that the Be/Xray binaries (Be-XRBs) are observed in regions with star-formation $(\mathrm{SF})$ rate bursts $\sim 30$ $70 \mathrm{Myr}$ ago, which coincides with the age of maximum Be-star formation, while regions with strong but more recent SF (e.g., the Wing) are deficient in Be-XRBs. Using the 2dF spectrograph of the Anglo-Australian Telescope (AAT) we have obtained optical spectra of 20 High-Mass Xray Binaries (HMXBs) in the SMC. All of these sources were proved to be Be-XRBs. Similar spectral-type distributions of Be-XRBs and Be field stars in the SMC have been found. On the other hand, the Be-XRBs in the Galaxy follow a different distribution than the isolated Be stars in the Galaxy, in agreement with previous studies.
\end{abstract}

Keywords. stars: emission-line, Be, stars: formation, galaxies: individual (SMC), Magellanic Clouds, X-rays: binaries

\section{Introduction: why observe the SMC?}

The SMC is the best target to study a, as complete as possible, XRB population. Similar studies in the Galaxy are hampered by extinction and distance uncertainties. The Large Magellanic Cloud (LMC) is much more extended than the SMC, requiring large area coverage to obtain sufficient numbers of XRBs, while other Local Group galaxies are too far to reach the quiescent population of HMXBs (typical $\mathrm{L}_{\mathrm{X}} \sim 10^{32-34} \mathrm{erg} \mathrm{s}^{-1}$; van Paradijs \& McClintock 1995). This way we are able to construct the luminosity function of the HMXBs in the central region of the SMC (see Zezas et al., these proceedings), and compare these luminosity functions with state of the art XRB synthesis models (e.g., Belczyński et al. 2008) and luminosity functions in other star-forming galaxies. In addition, the SMC hosts a large number of HMXBs (e.g., Haberl \& Pietsch 2004; McBride et al. 2008; Antoniou et al. 2008a).

\section{X-ray study of the SMC}

Using the ACIS-I detector on board Chandra we observed 5 fields (P.I. A. Zezas) in the central part of the SMC (the so called SMC "bar"), with typical exposure times of 8 $12 \mathrm{ks}$. These observations yielded a total of 158 sources, down to a limiting luminosity of $\sim 4 \times 10^{33} \mathrm{erg} \mathrm{s}^{-1}$ (in the $0.7-10 \mathrm{keV}$ band), reaching the luminosity range of quiescent HMXBs. The analysis of the data, the source-list and their X-ray luminosity functions (XLFs) are presented in Zezas et al. (in preparation), while their optical counterparts and resulting classification are presented in Antoniou et al. (2008a). 
Our XMM-Newton survey (P.I. A. Zezas) consists of 5 observations in the outer parts of the SMC, performed with the 3 EPIC (MOS1, MOS2 and PN) detectors in the full frame mode. The observed fields were selected to sample stellar populations in a range of ages ( 10-500 Myr; based on the SF history of Harris \& Zaritsky 2004). One of these fields was affected by high background flares and it is not included in the current study. We detected 186 sources down to a limiting luminosity of $\sim 3.5 \times 10^{33} \mathrm{erg} \mathrm{s}^{-1}$ in the $0.2-12 \mathrm{keV}$ band. More details on the data analysis and the final source-list (including the XLFs) will be presented in Antoniou et al. (in preparation).

\section{Candidate Be-XRBs}

In order to identify the Be-XRBs that lie in our fields we first study the X-ray properties of the sources. Be-XRBs show pulsations and have hard 1-10 keV spectra (i.e. with a power-law energy index of $\Gamma<1.6$; e.g., Yokogawa et al. 2003), which are signatures of accretion onto strongly magnetized neutron stars. This information is derived from X-ray spectral fits. However, for sources with small number of counts we use X-ray color-color diagrams (e.g., Prestwich et al. 2003).

The next step is the identification of an early (O or B) type star as the optical counterpart of these selected hard X-ray sources. We cross-correlate their position with optical photometric catalogs within a search radius calculated from the combination, in quadrature, of the astrometric uncertainty of the corresponding optical catalog, and the positional uncertainty for each X-ray source. In the present work, we have used two optical catalogs: the OGLE-II (Udalski et al. 1998) and the Magellanic Clouds Photometric Survey (MCPS; Zaritsky et al. 2002). In crowded fields, actual photometric (as well astrometric) uncertainties can be larger, due to source confusion, which we consider to be more severe in the MCPS catalog, due to the larger pixel size, and worse overall seeing. However, because of the incomplete coverage of the Chandra and XMM-Newton fields by the OGLE-II survey ( $\sim 70 \%$ and $<40 \%$, respectively), we supplemented the optical data with the MCPS catalog, which fully covers the observed fields.

In order to identify an early-type counterpart, we use the locus of early (O and B) type stars in the $V, B-V$ color-magnitude diagram. We define this locus by using data from the $2 \mathrm{dF}$ spectroscopic survey of SMC stars (the most extended such catalog available; Evans et al. 2004). In addition, we perform Monte-Carlo simulations and we estimate the chance-coincidence probability for the $\mathrm{O}$ or B type stars to be $\leqslant 19 \%$ for the Chandra fields and $\sim 8 \%$ for the XMM-Newton fields.

Following the above approach, we find 9 and 7 new candidate Be-XRBs within the Chandra and XMM-Newton fields, respectively. Moreover, our results are consistent with previous classifications in all cases of overlap (18 for the Chandra and 1 for the XMMNewton in total; all Be-XRBs). If we add to the above numbers the confirmed and candidate Be-XRBs that lie in our fields but have not been detected in our surveys (from the latest census of Magellanic Clouds HMXBs of Liu et al. 2005), we have a total of 29 and 9 Be-XRBs in the Chandra and XMM-Newton fields, respectively. We note that because of the transient nature of these systems, their numbers can be considered only as lower limits, but are nonetheless representative of the relative populations in the observed fields.

\section{The "overabundance" of SMC Be-XRBs with respect to the Galaxy}

It is widely accepted that the SMC hosts an unusual large number of HMXBs and Be-XRBs when compared to the Galaxy (see contribution by Coe, this volume). In order 
to investigate this, we have to minimize the age effects or variations due to SF rate differences for populations of different ages. This is feasible by studying the Be-XRBs with respect to their related stellar populations, i.e. the ratio of Be-XRBs to OB stars within an area. For the Be-XRBs in the Chandra SMC fields and the Galaxy we used sources with an X-ray luminosity limit of $\sim 10^{34} \mathrm{erg} \mathrm{s}^{-1}$, while for the Galaxy we only kept sources within $10 \mathrm{kpc}$ of the Sun. For the Galactic HMXBs we used the compilation of Liu et al. (2006), while for the SMC we supplemented the catalog of Liu et al. (2005) with our candidate SMC Be-XRBs (see $\S 3$ ). The OB stars for the SMC fields are derived from the MCPS catalog (based on the $V$ magnitude and $B-V$ color), while for the Galaxy we used the compilation of Reed (2001).

We find that Be-XRBs are $\sim 2$ times more common in the SMC when compared to the Galaxy, thus there is still a residual excess that cannot be accounted for by the difference in the SF rate. However, this residual excess can be attributed to the lower metallicity of the $\mathrm{SMC}\left(\sim 0.2 \mathrm{Z}_{\odot}\right)$. Population synthesis models predict a factor of $\sim 3$ higher numbers of HMXBs in galaxies with metallicities similar to that of the SMC, when compared to the Galaxy (Dray 2006). In addition there is observational evidence for a trend of higher proportion of Be stars in lower metallicity environments (at least in the case of younger systems - Wisniewski \& Bjorkman 2006; Martayan et al. 2007). We thus conclude that on its own, neither the lower SMC metallicity nor the enhanced SF rate at the age of $\sim 40 \mathrm{Myr}$ ago, can produce the observed 'overabundance' of SMC Be-XRBs (see also Antoniou et al. 2008a and McBride et al. 2008).

\section{SMC X-ray source populations as a function of age}

The SMC "bar" hosts young stellar populations (typically < $100 \mathrm{Myr}$; e.g., Harris \& Zaritsky 2004), and the vast majority of SMC pulsars (Galache et al. 2008). Shtykovskiy \& Gilfanov (2007) found that the age distribution of the HMXBs peaks at $~ 20-50$ Myr after the SF event, while McSwain \& Gies (2005) observed a strong evolution in the fraction of Be stars with age up to $100 \mathrm{Myr}$, with a maximum at $7.4<\log ($ age $)<7.8$. These results motivated us to investigate the connection of the SF history of our Chandra and XMM-Newton fields with their population of Be-XRBs.

Using data from Harris \& Zaritsky (2004) we derive the recent SF history in our Chandra and XMM-Newton fields, by calculating the average SF history of the MCPS regions $\left(\sim 12^{\prime} \times 12^{\prime}\right)$ encompassed by them. We find that:

(i) For the Chandra fields, the most recent major burst peaked $\sim 42 \mathrm{Myr}$ ago, and it had a duration of $\sim 40$ Myr. In addition, there were older SF episodes $(\sim 0.4$ Gyr ago $)$ with lower intensity but longer duration, as well as a more recent episode $(\sim 11 \mathrm{Myr})$ observed only in one of the Chandra fields.

(ii) For one of our XMM-Newton fields, the most recent major burst occurred $\sim 67 \mathrm{Myr}$ ago. We also observed two fields with very young populations (most recent major burst at $\sim 11$ and $\sim 17$ Myr ago, respectively). Both these fields have additional less intense bursts $\sim 67$ Myr ago.

\subsection{Link between $S F$ and the XRB populations}

In order to investigate the link between SF and the XRB populations, we calculate the average SF history for the MCPS regions $\left(\sim 12^{\prime} \times 12^{\prime}\right.$; Harris \& Zaritsky 2004$)$ that host one or more Be-XRBs (candidate and confirmed) detected in our Chandra and XMMNewton surveys. The SF history at each region is weighted by the encompassed number of Be-XRBs. We repeat this exercise for the MCPS regions that do not have any known Be-XRB in our surveys. The two SF histories are presented in Fig.1 (upper panel). 


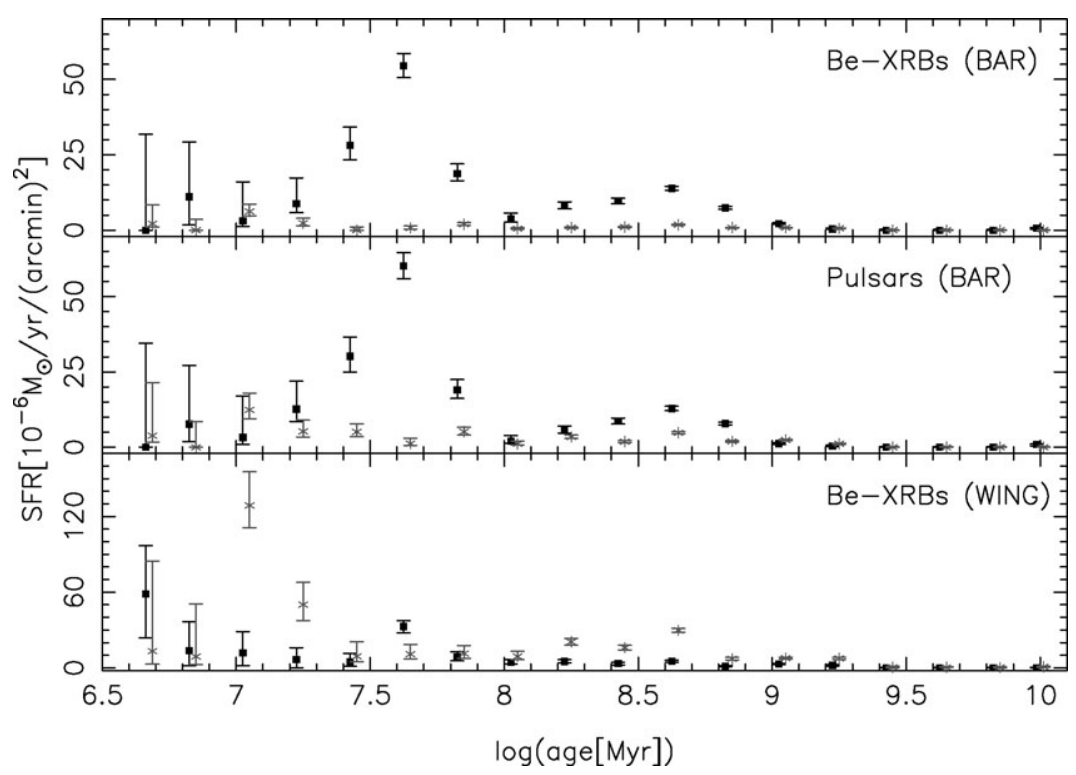

Figure 1. (upper panel) The average SF history for the MCPS regions (using data from Harris \& Zaritsky 2004) which overlap with our Chandra and XMM-Newton fields and host one or more (shown in black) or none (shown in gray) detected Be-XRBs (candidate and confirmed). (middle panel) The same plot as above but for MCPS regions with and without X-ray pulsars, and (bottom panel) with and without Be-XRBs from the Chandra Wing survey (P.I. Coe, AO6). For clarity a small offset of $\log ($ age $[\mathrm{Myr}]) \sim 0.025$ has been applied in the distributions of areas without Be-XRBs and/or pulsars.

Following the above comparison, we construct (middle panel of Fig.1) the SF history for the MCPS regions (overlapping with any of our fields) that host one or more known X-ray pulsars $\dagger$ (shown in black), and for those that do not host such sources (shown in gray). A large fraction of these pulsars also appears in the Be-XRBs sample, since most of the companions of the SMC pulsars are Be stars. However, for completeness we present both (upper and middle panel, respectively), since the pulsars are X-ray selected while the Be-XRBs used above are selected based on the optical properties of the companion stars. In total, in our Chandra fields lie 19 X-ray pulsars, while in the XMM-Newton fields only 3. As expected, the pattern in their SF history is very similar to that of Be-XRBs. In Fig.1 (bottom panel) we also present the average SF history for the MCPS regions with any (shown in black) and without (shown in grayq) Be-XRBs detected in the Chandra Wing survey (P.I. M. Coe, AO6). This survey covered 20 fields, however 3 of those were not used here because they do not overlap with any MCPS region, while all 4 Be-XRBs are also X-ray pulsars (Schurch et al. 2007).

From the above analysis we find that the number of Be-XRBs peaks for stellar populations of ages $\sim 30-70$ Myr. This is consistent with the study of McSwain \& Gies (2005), who find that Be stars develop their decretion disks at ages of $\sim 25-80 \mathrm{Myr}$, with a peak at $\sim 35 \mathrm{Myr}$. OB stars formed during this episode are expected to reach the maximum rate of decretion disk formation at the current epoch. We also find that the other two peaks ( $\sim 11$ and $\sim 422 \mathrm{Myr}$ ) observed in the SF history of regions with Be-XRBs do not give any Be-XRBs as expected. The first one (at $\sim 11 \mathrm{Myr}$ ) is too early to give any pulsar

$\dagger$ Using the on-line census of Malcolm Coe (http://www.astro.soton.ac.uk/ mjc/ as of 06/05/2007). 


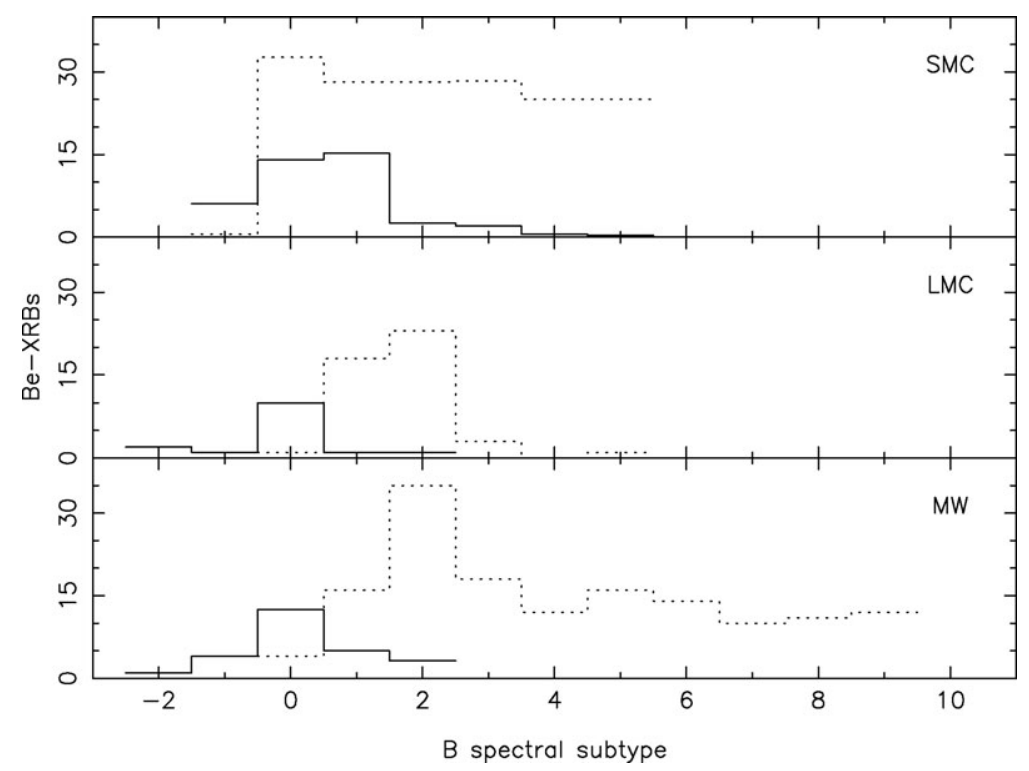

Figure 2. Comparison of the B spectral subtype distributions of Be-XRBs (solid histograms) to isolated Be stars (dashed histograms) in the SMC (top panel), the LMC (middle panel), and the Milky Way (bottom panel). Negative spectral subtypes correspond to O-type stars.

Be-XRB, while the second SF rate peak (at $\sim 422 \mathrm{Myr}$ ) cannot result in Be-XRB formation, since by that time all OB stars have become supernovae. The peak at $\sim 422 \mathrm{Myr}$ ago is also observed in the global SF history of the SMC, and it temporally coincides with past perigalactic passages of the SMC with the Galaxy (Harris \& Zaritsky 2004). A similar study by Shtykovskiy \& Gilfanov (2007), reached the same conclusions, however these authors did not attribute the large number of SMC Be-XRBs to the timescale of maximum Be star formation.

Furthermore, the lack of a large number of Be-XRBs in the SMC Wing is consistent with the present study. As it is shown in Fig. 1 (bottom panel), the Wing has a weaker than the "bar" SF burst at the age of enhanced formation of Be stars (i.e. at $\sim 42 \mathrm{Myr}$ ), while its most recent intense SF burst occurred only $\sim 11$ Myr ago. Thus we do not expect a significant number of SMC Wing Be-XRBs, at least comparable to that in the SMC 'bar'. Since in the present study we used the number of Be-XRBs from a single observation of each field and for fields covering both the SMC "bar" and the Wing, we were able to minimize the effects of the transient Be-XRB nature.

\section{Optical spectroscopy of $20 \mathrm{SMC}$ Be-XRBs}

Using the 2dF spectrograph of the AAT we observed 20 HMXBs (Antoniou et al. 2008b) detected with Chandra (Zezas et al., in prep.) and XMM-Newton (Haberl \& Pietsch 2004). All of these sources were proved to be Be-XRBs. The spectral classification of 6 previously classified Be-XRBs have been revisited, while we estimate that our spectral types are accurate to better than \pm 1 subclass in most cases, especially for earlier than B2 spectral types.

The distribution of spectral types of our Be-XRB sample shows a peak at B1.5. In Figure 2 we present the B spectral subtype distributions of Be-XRBs (solid histograms) and of isolated Be stars (dashed histograms) in the SMC (top panel), the LMC (middle panel), and the Galaxy (bottom panel). Negative spectral subtypes correspond to O-type 
stars. Whenever only a broad spectral class was available, we equally divided the contribution in the different subtypes. We find similar spectral-type distributions of Be-XRBs and Be field stars in the SMC. On the other hand, the Be-XRBs in the Galaxy follow a different distribution than the isolated Be stars in the Galaxy, in agreement with previous studies.

This work also reinforces the $\mathrm{P}_{\text {orb }}-\mathrm{H} \alpha$ equivalent-width relation that holds for BeXRBs. As Reig (2007) explained, the neutron star does not allow the companion star to develop a large decretion disc in cases of small orbital period systems, thus its presence leads to the tidal truncation of the disc, and this in turn to smaller $\mathrm{H} \alpha \mathrm{EW}$ values. This is the first such study which demonstrates the importance of the Be-XRBs as a dominant component of young XRB populations.

\section{Acknowledgements}

We would like to thank Nolan Walborn for fruitful discussions on the spectral classification. VA acknowledges support from Marie Curie grant no. 39965 to the Foundation for Research and Technology - Hellas, NASA LTSA grant NAG5-13056, and NASA grant GO2-3117X.

\section{References}

Antoniou, V., Zezas, A., Hatzidimitriou, D., \& McDowell, J. 2008a, ApJ, submitted

Antoniou, V., Hatzidimitriou, D., Zezas, A., \& Reig, P. 2008b, ApJ, submitted

Belczyński, K., Kalogera, V., Rasio, F. A., Taam, R. E., Zezas, A., Bulik, T., Maccarone, T. J., \& Ivanova, N. 2008, ApJS, 174, 223

Dray, L. M. 2006, MNRAS, 370, 2079

Evans, C. J., Howarth, I. D., Irwin, M. J., Burnley, A. W., \& Harries, T. J. 2004, MNRAS, 353, 601

Galache, J. L., Corbet, R. H. D., Coe, M. J., Laycock, S., Schurch, M. P. E., Markwardt, C., Marshall, F. E., \& Lochner, J. 2008, ApJS, 177, 189

Haberl, F. \& Pietsch, W. 2004, A\& A, 414, 667

Harris, J. \& Zaritsky, D. 2004, AJ, 127, 1531

Liu, Q. Z., van Paradijs, J., \& van den Heuvel, E. P. J. 2005, A\&SA, 442, 1135

Liu, Q. Z., van Paradijs, J., \& van den Heuvel, E. P. J. 2006, $A \& A$, 455, 1165

Martayan, C., Frémat, Y., Hubert, A. -M., Floquet, M., Zorec, J., \& Neiner, C. 2007, A\&A, 462,683

McBride, V. A., Coe, M. J., Negueruela, I., Schurch, M. P. E., \& McGowan, K. E. 2008, MNRAS, 388,1198

McSwain, M. V. \& Gies, D. R. 2005, ApJS, 161, 118

Prestwich, A. H., Irwin, J. A., Kilgard, R. E., Krauss, M. I., Zezas, A., Primini, F., Kaaret, P., \& Boroson, B. 2003, ApJ, 595, 719

Reed, B. C. 2001, PASP, 113, 537

Reig, P. 2007, MNRAS, 377, 867

Schurch, M. P. E., Coe, M. J., McGowan, K. E., et al. 2007, MNRAS, 381, 1561

Shtykovskiy, P. E. \& Gilfanov, M. R. 2007, Astron. Lett., 33, 437

Udalski, A., Szymański, M., Kubiak, M., Pietrzyński, G., Wozniak, P., \& Żebruń, K. 1998, AcA, 48,147

van Paradijs, J. \& McClintock, J. E. 1995, in W. H. G. Lewin, J. van Paradijs, \& E. P. J. van den Heuvel (eds.), X-ray Binaries (Cambridge: CUP), p. 58

Wisniewski, J. P. \& Bjorkman, K. S. 2006, ApJ, 652, 458

Yokogawa, J., Imanishi, K., Tsujimoto, M., Koyama, K., \& Nishiuchi, M. 2003, PASJ, 55, 161

Zaritsky, D., Harris, J., Thompson, I. B., Grebel, E. K., \& Massey, P. 2002, AJ, 123, 855 